\title{
Shell Polynomials and Dual Birth-Death Processes ${ }^{\star}$
}

\author{
Erik A. VAN DOORN
}

Department of Applied Mathematics, University of Twente, P.O. Box 217, 7500 AE Enschede, The Netherlands

E-mail: e.a.vandoorn@utwente.nl

URL: http://wwwhome.math.utwente.nl/ doornea/

Received January 02, 2016, in final form May 14, 2016; Published online May 18, 2016

http://dx.doi.org/10.3842/SIGMA.2016.049

\begin{abstract}
This paper aims to clarify certain aspects of the relations between birth-death processes, measures solving a Stieltjes moment problem, and sets of parameters defining polynomial sequences that are orthogonal with respect to such a measure. Besides giving an overview of the basic features of these relations, revealed to a large extent by Karlin and McGregor, we investigate a duality concept for birth-death processes introduced by Karlin and McGregor and its interpretation in the context of shell polynomials and the corresponding orthogonal polynomials. This interpretation leads to increased insight in duality, while it suggests a modification of the concept of similarity for birth-death processes.
\end{abstract}

Key words: orthogonal polynomials; birth-death processes; Stieltjes moment problem; shell polynomials; dual birth-death processes; similar birth-death processes

2010 Mathematics Subject Classification: 42C05; 60J80; 44A60

\section{Introduction}

In what follows a measure will always be a finite positive Borel measure on the real axis with infinite support and finite moments of all (positive) orders. It will be convenient to assume throughout that the measure is normalized so that it becomes a probability measure. The Hamburger moment problem associated with a measure $\psi$ is said to be determined $(\psi$ is $\operatorname{det}(\mathrm{H})$, for short) if $\psi$ is uniquely determined by its moments; otherwise, it is said to be indeterminate $(\psi$ is indet $(\mathrm{H}))$. Similar terminology will be used for the Stieltjes moment problem associated with $\psi$, in which we limit our scope to measures with support on the nonnegative real axis, with $\operatorname{det}(\mathrm{S})(\operatorname{indet}(\mathrm{S}))$ replacing $\operatorname{det}(\mathrm{H})(\operatorname{indet}(\mathrm{H}))$.

Chihara [9] showed that when a measure is $\operatorname{indet}(\mathrm{H})$ and has left-bounded support, there is a unique solution of the associated moment problem with the property that the minimum of its support is maximal. We will refer to this solution as the natural solution. It will be convenient to qualify a measure as natural also if it is the solution of a determined moment problem. Note that the natural solution of an indeterminate Hamburger moment problem may be the unique solution of a Stieltjes moment problem.

Our point of departure is a measure $\psi$ on the nonnegative real axis with moments

$$
m_{n}(\psi):=\int_{[0, \infty)} x^{n} \psi(d x), \quad n \geq 0 .
$$

By assumption $m_{0}(\psi)=1$. In what follows we allow $\psi$ to be indet(S) (and hence indet $(\mathrm{H})$ ) but assume in this case that $\psi$ is the natural solution of the associated moment problem. The (monic) polynomials that are orthogonal with respect to $\psi$ will be denoted by $P_{n}$.

\footnotetext{
${ }^{\star}$ This paper is a contribution to the Special Issue on Orthogonal Polynomials, Special Functions and Applications. The full collection is available at http://www.emis.de/journals/SIGMA/OPSFA2015.html
} 
As is well known there exist unique constants $c_{n} \in \mathbb{R}$ and $d_{n+1}>0, n \geq 1$, such that the polynomials $P_{n}$ satisfy the three-terms recurrence relation

$$
\begin{aligned}
& P_{n}(x)=\left(x-c_{n}\right) P_{n-1}(x)-d_{n} P_{n-2}(x), \quad n>1, \\
& P_{1}(x)=x-c_{1}, \quad P_{0}(x)=1 .
\end{aligned}
$$

Since the support of $\psi$ is a subset of the nonnegative real axis we actually have $c_{n}>0$, and there is a more refined result (see, for instance, Chihara [10, Corollary to Theorem I.9.1]) to the effect that there are numbers $\mu_{0} \geq 0$ and $\lambda_{n}>0, \mu_{n+1}>0$ for $n \geq 0$, such that

$$
c_{n}=\lambda_{n-1}+\mu_{n-1} \quad \text { and } \quad d_{n+1}=\lambda_{n-1} \mu_{n}, \quad n \geq 1 .
$$

(Further results in this vein can be found in [14].) Since $\lambda_{n}$ and $\mu_{n}$ may be interpreted as the birth rates and death rates, respectively, of a birth-death process on the nonnegative integers, we will refer to a collection of such constants as a set of birth and death rates (or a rate set, for short). More information on birth-death processes and their rates will be given in later sections, but at this stage we note that, by Karlin and McGregor [16, Lemma 1 and Lemma 6 on p. 527] (see also [14, Theorem 1.3]), we must have $\mu_{0}=0$ unless $\psi$ has a finite moment of order -1 , that is,

$$
m_{-1}(\psi):=\int_{[0, \infty)} x^{-1} \psi(d x)<\infty,
$$

in which case $\mu_{0}$ may be any number in the interval $\left[0,1 / m_{-1}(\psi)\right]$. Evidently, once $\mu_{0}$ has been chosen, the other rates are fixed.

In the remainder of this section we will assume that (1.3) is satisfied, so that, in particular, $\psi(\{0\})=0$. Defining the measure $\phi^{(0)}$ by

$$
\phi^{(0)}([0, x]):=\frac{1}{m_{-1}(\psi)} \int_{[0, x]} y^{-1} \psi(d y), \quad x \geq 0,
$$

and letting, for $a>0$,

$$
\phi^{(a)}:=\frac{1}{a+1}\left(a \delta_{0}+\phi^{(0)}\right)
$$

where $\delta_{0}$ is the Dirac measure with mass 1 at 0 , we observe that, for any $a \geq 0, \phi^{(a)}$ is a probability measure on the nonnegative real axis. As a consequence there exists a sequence of (monic) polynomials $\left\{S_{n}^{(a)}\right\}$ that are orthogonal with respect to $\phi^{(a)}$. Since, for all $a \geq 0$,

$$
\psi([0, x])=(a+1) m_{-1}(\psi) \int_{[0, x]} y \phi^{(a)}(d y), \quad x \geq 0,
$$

we will, following Chihara [13], refer to the polynomials $S_{n}^{(a)}$ as shell polynomials corresponding to the orthogonal polynomial sequence $\left\{P_{n}\right\}$. In the terminology of [10, Section I.7] the polynomials $P_{n}$ are, for any $a \geq 0$, the kernel polynomials with $K$-parameter 0 corresponding to $\left\{S_{n}^{(a)}\right\}$.

Information on the status of the moment problem associated with $\phi^{(a)}$ is given in the next theorem.

Theorem 1.1 (Chihara [8, Theorem 2]).

(i) The measure $\phi^{(0)}$ is $\operatorname{det}(\mathrm{H})$.

(ii) The measure $\phi^{(a)}, a>0$, is $\operatorname{det}(\mathrm{S})$ if and only if the measure $\psi$ is $\operatorname{det}(\mathrm{S})$. 
Evidently, $\phi^{(a)}$ cannot be a natural measure if it is indet(S), but we will see that $\phi^{(a)}$ nevertheless has a certain significance in this case, in particular in the context of birth-death processes. Before introducing our findings it will be useful to state already some facts about the relations between birth-death processes, rate sets, and measures that will be expounded in Section 3 .

Fact 1.2. A birth-death process uniquely defines a rate set.

Fact 1.3. A birth-death process uniquely defines a measure on $[0, \infty)$ through Karlin and McGregor's representation formula (3.3) for the transition functions of a birth-death process. This measure is natural or of a type known as Nevanlinna extremal.

Fact 1.4. A rate set $\left\{\lambda_{n}, \mu_{n}\right\}$ uniquely defines, through (1.2), a natural measure on $[0, \infty)$ with respect to which the polynomials $P_{n}$ of (1.1) are orthogonal. Conversely, a natural measure on $[0, \infty)$, with monic orthogonal polynomials $P_{n}$ and moment $m_{-1}$ of order -1 , defines, through (1.1) and (1.2), a rate set $\left\{\lambda_{n}, \mu_{n}\right\}$, which, if $m_{-1}=\infty$, is unique and satisfies $\mu_{0}=0$. If $m_{-1}<\infty$ the measure defines an infinite family of rate sets indexed by the value of $\mu_{0}$, which can be any number in the interval $\left[0,1 / m_{-1}\right]$.

Fact 1.5. A rate set $\left\{\lambda_{n}, \mu_{n}\right\}$ uniquely defines a birth-death process if and only if at least one of the following conditions prevails:

(i) the natural measure defined by the rate set is $\operatorname{det}(\mathrm{S})$;

(ii) $\mu_{0}>0$ and the natural measure defined by the rate set has $m_{-1}=\mu_{0}^{-1}$.

Otherwise, there is an infinite, one-parameter family of birth-death processes with the given rates. Two members of this family may be identified as extreme, and are known as the minimal process (associated with the natural measure) and the maximal process.

Fact 1.2 is a trivial consequence of the definition of a birth-death process in Section 3.1; Facts 1.3 and 1.4 follow from the seminal work of Karlin and McGregor [16] (Fact 1.4 also summarizes earlier observations in this section); for Fact 1.5 we refer to [16] again and, for the second part, to [22]. Note that, by Fact 1.4, our measure $\psi$ defines infinitely many rate sets, since $m_{-1}(\psi)<\infty$ by assumption.

Our main goal in this paper is to interpret and characterize the measures $\phi^{(a)}, a \geq 0$, and the relation between the measures $\psi$ and $\phi^{(a)}$, in the context of birth-death processes. Concretely, we will display a one-to-one correspondence between the measures $\phi^{(a)}, a \geq 0$, and the rate sets with $\mu_{0}>0$ defined by $\psi$ (to which we will refer as $\psi$-rate sets for short). Moreover, if $\phi^{(a)}$ is $\operatorname{det}(\mathrm{S})$, then the birth-death process (uniquely) defined by $\phi^{(a)}$ and the (unique) birth-death process whose rate set is the $\psi$-rate set corresponding to $\phi^{(a)}$ are shown to be dual to each other in sense of Karlin and McGregor [17, Section 6]. As a result the duality concept for birth-death processes can be extended to families of birth-death processes that are similar in the sense of [18], after a slight modification of the definition of similarity.

When $\phi^{(a)}$ is indet(S) - and hence, by Theorem 1.1, $a>0$ - the situation is more complicated since the duality concept for birth-death processes can be applied only to minimal and maximal processes when a rate set does not define a birth-death process uniquely (see [22]). However, we will see that in this case $\phi^{(a)}$ is Nevanlinna extremal (as stated already by Berg and Christiansen [3]) and corresponds to a maximal birth-death process, which happens to be dual to the minimal process whose rate set is the $\psi$-rate set corresponding to $\phi^{(a)}$. We will also present a counterpart of this result.

In the next section we will collect some further notation, terminology and preliminary results about shell polynomials, rate sets, and measures, while in Section 3 the relevant properties of birth-death processes are set forth and put in proper perspective. Our findings are detailed in Section 4 . 


\section{Preliminaries}

\subsection{Shell polynomials and rate sets}

Applying [10, Corollary to Theorem I.9.1] to the polynomials $S_{n}^{(a)}$, we conclude that, for any $a \geq 0$, there exist constants $\mu_{0}^{(a)} \geq 0$ and $\lambda_{n}^{(a)}>0, \mu_{n+1}^{(a)}>0$ for $n \geq 0$, such that

$$
\begin{aligned}
& S_{n}^{(a)}(x)=\left(x-\lambda_{n-1}^{(a)}-\mu_{n-1}^{(a)}\right) S_{n-1}^{(a)}(x)-\lambda_{n-2}^{(a)} \mu_{n-1}^{(a)} S_{n-2}^{(a)}(x), \quad n>1, \\
& S_{1}^{(a)}(x)=x-\lambda_{0}^{(a)}-\mu_{0}^{(a)}, \quad S_{0}^{(a)}(x)=1 .
\end{aligned}
$$

If $\phi^{(a)}$ is natural and $m_{-1}\left(\phi^{(a)}\right)=\infty$ (so in particular if $\phi^{(a)}$ is $\operatorname{det}(\mathrm{S})$ and $a>0$ ) then, by [16, Lemma 1 and Lemma 6 on p. 527)] again, we must have $\mu_{0}^{(a)}=0$. In other circumstances $\mu_{0}^{(a)}$ may also be chosen positive, but it will be convenient to set $\mu_{0}^{(a)}=0$ by definition in what follows.

We can now relate the parameters in the recurrence relation $(2.1)$ for the polynomials $S_{n}^{(a)}$ to the parameters $c_{n}$ and $d_{n}$ in the recurrence relation (1.1). Indeed, since the polynomials $P_{n}$ are the kernel polynomials with $K$-parameter 0 corresponding to $\left\{S_{n}^{(a)}\right\}$, we have, by [10, Theorem I.9.1],

$$
c_{n}=\lambda_{n-1}^{(a)}+\mu_{n}^{(a)} \quad \text { and } \quad d_{n+1}=\lambda_{n}^{(a)} \mu_{n}^{(a)}, \quad n \geq 1 .
$$

Subsequently defining birth rates $\lambda_{n}$ and death rates $\mu_{n}$ by

$$
\lambda_{n}=\mu_{n+1}^{(a)} \quad \text { and } \quad \mu_{n}=\lambda_{n}^{(a)}, \quad n \geq 0,
$$

it follows that we have regained (1.2). So we see that we can parametrize the birth and death rates in the representation (1.2) by the value of $\mu_{0}$, but also by the size $a$ of the atom at 0 of the measure $\phi^{(a)}$ of (1.4) and (1.5), since the value of $a$ uniquely identifies the shell polynomials $S_{n}^{(a)}$ corresponding to $\left\{P_{n}\right\}$, and hence, through (2.1) (where $\left.\mu_{0}^{(a)}=0\right)$ and (2.2), the birth and death rates.

The next theorem gives an explicit one-to-one relation between $\mu_{0}$ and $a$, and shows that the question of whether the alternative representation yields all possibilities can be answered in the affirmative, provided we allow $0 \leq a \leq \infty$ and interpret $\lambda_{n}^{(\infty)}$ and $\mu_{n}^{(\infty)}$ as limits as $a \rightarrow \infty$ of the corresponding quantities with superindex ${ }^{(a)}$. We will see in Section 2.2 that the theorem is an immediate corollary of a theorem of Chihara [8].

Theorem 2.1. Let $\psi$ be a natural measure satisfying (1.3) and let $\mu_{0}$ be determined by a via (1.4), (1.5), (2.1) (with $\left.\mu_{0}^{(a)}=0\right)$ and (2.2). Then, for $0 \leq a \leq \infty$,

$$
\mu_{0}=\frac{1}{(a+1) m_{-1}(\psi)},
$$

whence $\mu_{0}$ can have any value in the interval $\left[0,1 / m_{-1}(\psi)\right]$.

Note that, as $a \rightarrow \infty, \phi^{(a)}$ converges strongly to $\delta_{0}$, so we cannot (and need not) extend the definition of $S_{n}^{(a)}$ to include the case $a=\infty$. An interpretation of $\lambda_{n}^{(\infty)}$ and $\mu_{n}^{(\infty)}$ as birth and death rates of a birth-death process on the nonnegative integers is possible, but does not fit in the setting described around (1.2) since $\lambda_{0}^{(\infty)}=\mu_{0}^{(\infty)}=0$.

Let us mention at this point that (2.2) displays the duality concept for birth-death processes that will be further discussed in Section 3.2 and plays a crucial role in Section 4 . 


\subsection{Chain sequences and rate sets}

We first recall some definitions and basic results (see Chihara [10, Section III.5] and [12] for more information). A sequence $\left\{a_{n}\right\}_{n=1}^{\infty}$ is a chain sequence if there exists a second sequence $\left\{g_{n}\right\}_{n=0}^{\infty}$ such that

(i) $0 \leq g_{0}<1, \quad 0<g_{n}<1, \quad n \geq 1$,

(ii) $\quad a_{n}=\left(1-g_{n-1}\right) g_{n}, \quad n \geq 1$.

The sequence $\left\{g_{n}\right\}$ is called a parameter sequence for $\left\{a_{n}\right\}$. If both $\left\{g_{n}\right\}$ and $\left\{h_{n}\right\}$ are parameter sequences for $\left\{a_{n}\right\}$, then

$$
g_{n}<h_{n}, \quad n \geq 0 \quad \Longleftrightarrow \quad g_{0}<h_{0} .
$$

Every chain sequence $\left\{a_{n}\right\}$ has a minimal parameter sequence, uniquely determined by the condition $g_{0}=0$, and a maximal parameter sequence $\left\{M_{n}\right\}$, characterized by the fact that $M_{0}>g_{0}$ for any other parameter sequence $\left\{g_{n}\right\}$. For every $x, 0 \leq x \leq M_{0}$, there is a unique parameter sequence $\left\{g_{n}\right\}$ for $\left\{a_{n}\right\}$ such that $g_{0}=x$.

Linking the parameters in the three-terms recurrence relation (1.1) to birth and death rates is an alternative for the approach involving chain sequences chosen by Chihara in, for instance, $[8,10]$. Indeed, letting

$$
a_{n}=\frac{d_{n+1}}{c_{n} c_{n+1}}, \quad n \geq 1,
$$

we see that the sequence $\left\{a_{n}\right\}_{n=1}^{\infty}$ is a chain sequence, since $a_{n}=\left(1-g_{n-1}\right) g_{n}$ if we choose

$$
g_{n}=\frac{\mu_{n}}{\lambda_{n}+\mu_{n}}, \quad n \geq 0
$$

for any set of birth rates $\lambda_{n}$ and death rates $\mu_{n}$ satisfying (1.2). So (2.3) gives a one-toone correspondence between a parameter sequence for the chain sequence $\left\{a_{n}\right\}$ and a rate set satisfying (1.2). Since $0 \leq \mu_{0} \leq 1 / m_{-1}(\psi)$, we can also characterize the maximal parameter sequence for $\left\{a_{n}\right\}$ by

$$
M_{0}=\frac{1}{c_{1} m_{-1}(\psi)} \text {. }
$$

Invoking [8, Theorem 2] we can now conclude that it implies Theorem 2.1, for on comparing our (1.5) with [8, equation (3.3)] and making appropriate identifications, we find that $a=$ $\left(\mu_{0} m_{-1}(\psi)\right)^{-1}-1$, as required.

\subsection{Spectral properties and Nevanlinna extremal measures}

In this subsection we will introduce some notation and terminology concerning the natural measure $\psi$ introduced in Section 1 and, if $\psi$ is indet(S), related measures called Nevanlinna extremal.

Of interest to us will be the quantities $\xi_{i}$, recurrently defined by

$$
\xi_{1}:=\inf \operatorname{supp}(\psi)
$$

and

$$
\xi_{i+1}:=\inf \left\{\operatorname{supp}(\psi) \cap\left(\xi_{i}, \infty\right)\right\}, \quad i \geq 1 .
$$


where $\operatorname{supp}(\psi)$ denotes the support (or spectrum) of the measure $\psi$. We further define

$$
\sigma:=\lim _{i \rightarrow \infty} \xi_{i}
$$

the first accumulation point of $\operatorname{supp}(\psi)$ if it exists, and infinity otherwise. So ( $\operatorname{supp} \psi)$ is discrete with no finite limit point if and only if $\sigma=\infty$. It is clear from the definition of $\xi_{i}$ that, for all $i \geq 1$,

$$
\xi_{i+1} \geq \xi_{i} \geq 0
$$

and

$$
\xi_{i}=\xi_{i+1} \quad \Longleftrightarrow \quad \xi_{i}=\sigma .
$$

Note that we must have $\sigma=0$ if $\xi_{1}=0$ and $\psi(\{0\})=0$. Also, $\psi$ must be $\operatorname{det}(\mathrm{S})$ if $\xi_{1}=0$.

From Karlin and McGregor [16] (see also Chihara [11]) we know that

$$
\psi \quad \text { is } \operatorname{indet}(\mathrm{S}) \Longleftrightarrow \sum_{n=0}^{\infty}\left(\pi_{n}+\frac{1}{\lambda_{n} \pi_{n}}\right)<\infty,
$$

where

$$
\pi_{0}:=1 \quad \text { and } \quad \pi_{n}:=\frac{\lambda_{0} \lambda_{1} \cdots \lambda_{n-1}}{\mu_{1} \mu_{2} \cdots \mu_{n}}, \quad n \geq 1,
$$

and $\left\{\lambda_{n}, \mu_{n}\right\}$ is the rate set with $\mu_{0}=0$ satisfying (1.2). We note, parenthetically, that for a rate set with $\mu_{0}>0$ the right-hand side of (2.7) is sufficient, but not necessary for the corresponding natural measure to be indet(S) (see [11]).

It is well known that $\sigma=\infty$ if $\psi$ is indet(S). (A necessary and sufficient condition for $\sigma=\infty$ in terms of any rate set satisfying (1.2) has recently been revealed in [24].) Moreover, if $\psi$ is indet(S) there are infinitely many solutions of the Stieltjes moment problem associated with $\psi$. We shall be interested in particular in solutions known as Nevanlinna extremal (or $N$-extremal, for short), which may be defined as follows (see, for example, Berg and Valent [7, Section 1] and, for more background information, Shohat and Tamarkin [21, pp. 51-60] and Berg and Valent [6, Section 2]). Let

$$
\rho(x):=\left\{\sum_{n=0}^{\infty} p_{n}^{2}(x)\right\}^{-1}, \quad x \in \mathbb{R},
$$

where $p_{n}(x)$ are the orthonormal polynomials corresponding to $\psi$. Then $\rho(x)$ is positive for all real $x$ and equals, if $x \geq 0$, the maximal mass any solution can concentrate at $x$. Supposing that a solution of the Stieltjes moment problem locates positive mass at the point $x$, then that solution is an $N$-extremal solution if and only if the point mass at $x$ equals $\rho(x)$.

Some pertinent properties of $N$-extremal solutions are the following. There is a one-to-one correspondence between the real numbers in the interval $\left[0, \xi_{1}\right]$ and the $N$-extremal solutions of the Stieltjes moment problem associated with $\psi$. For $\xi \in\left[0, \xi_{1}\right]$ we denote the corresponding $N$ extremal solution by $\psi_{\xi}$. The spectrum of $\psi_{\xi}$ is discrete and consists of the point $\xi$ and exactly one point in each of the intervals $\left(\xi_{i}, \xi_{i+1}\right], i \geq 1$. Evidently, we have $\psi_{\xi_{1}}=\psi$ and $\operatorname{supp}\left(\psi_{\xi_{1}}\right)$ $=\left\{\xi_{1}, \xi_{2}, \ldots\right\}$. The spectral points of two different $N$-extremal solutions strictly separate each other.

We finally note that the natural measure $\psi$ can be identified with the solution of the associated Stieltjes moment problem that is related to the Friedrichs extension of some semi-bounded operator (see Pedersen [19] for details). The parametrization in [19] (and in [6, 7]) of the $N$-extremal solutions of the indeterminate Stieltjes moment problem associated with $\psi$ differs from ours and is effectuated by a number in an interval $[\alpha, 0]$; the solution corresponding to the parameter value $\alpha(<0)$ is our natural solution $\psi\left(=\psi_{\xi_{1}}\right)$. 


\section{Birth-death processes}

\subsection{Basic properties}

In this paper a birth-death process $\mathcal{X} \equiv\{X(t), t \geq 0\}$, say, will always be a continuous-time Markov chain taking values in $\mathcal{N}:=\{0,1, \ldots\}$ with the property that only transitions to neighbouring states are permitted. The process has upward transition (or birth) rates $\lambda_{n}, n \in \mathcal{N}$, and downward transition (or death) rates, $\mu_{n}, n \in \mathcal{N}$, all strictly positive except $\mu_{0}$, which might be equal to 0 . When $\mu_{0}=0$ the process is irreducible, but when $\mu_{0}>0$ the process may escape from $\mathcal{N}$, via 0 , to an absorbing state -1 . The $q$-matrix of transition rates of $\mathcal{X}$, restricted to the states in $\mathcal{N}$, will be denoted by $Q$, that is,

$$
Q=\left(\begin{array}{cccccc}
-\left(\lambda_{0}+\mu_{0}\right) & \lambda_{0} & 0 & 0 & 0 & \ldots \\
\mu_{1} & -\left(\lambda_{1}+\mu_{1}\right) & \lambda_{1} & 0 & 0 & \ldots \\
0 & \mu_{2} & -\left(\lambda_{2}+\mu_{2}\right) & \lambda_{2} & 0 & \ldots \\
\ldots & \ldots & \ldots & \ldots & \ldots & \ldots \\
\ldots & \ldots & \ldots & \ldots & \ldots & \ldots
\end{array}\right),
$$

and $\mathcal{X}$ will be referred to as a $Q$-process. The process $\mathcal{X}$ will be identified with its transition functions

$$
p_{i j}(t):=\operatorname{Pr}\{X(t)=j \mid X(0)=i\}, \quad i, j \in \mathcal{N}, \quad t \geq 0,
$$

and we write $P(\cdot):=\left(p_{i j}(\cdot), i, j \in \mathcal{N}\right)$. Besides the usual probabilistic requirements and the Chapman-Kolmogorov equations

$$
P(s+t)=P(s) P(t), \quad s \geq 0, \quad t \geq 0,
$$

imposed by the Markov property, the transition functions of $\mathcal{X}$ will be assumed to satisfy both the Kolmogorov backward equations

$$
P^{\prime}(t)=Q P(t), \quad t \geq 0
$$

and forward equations

$$
P^{\prime}(t)=P(t) Q, \quad t \geq 0,
$$

with initial condition $P(0)=I$, the identity matrix. It follows in particular that $P^{\prime}(0)=Q$, establishing Fact 1.2. We refer to Anderson [1] for more information on continuous-time Markov chains in general and birth-death processes in particular.

A matrix of the type (3.1) is always the $q$-matrix of a birth-death process, but not necessarily of a unique process. Karlin and McGregor [16] have shown that the $Q$-process $\mathcal{X}$ is uniquely determined by $Q$ - that is, by its rates - if and only if the series

$$
\sum_{n=0}^{\infty}\left(\pi_{n}+\frac{1}{\lambda_{n} \pi_{n}}\right) \text {, }
$$

where $\pi_{n}$ is given by (2.8), diverges. If $\mu_{0}=0$ then, in view of (2.7) (where $\mu_{0}=0$ is assumed), the series diverges if and only if $\psi$ is $\operatorname{det}(\mathrm{S})$, where $\psi$ denotes the (natural) measure defined by the rate set $\left\{\lambda_{n}, \mu_{n}\right\}$. If $\mu_{0}>0$ then, by [16, Theorem 15], the series (3.2) diverges if and only if $\psi$ is $\operatorname{det}(\mathrm{S})$ or $m_{-1}(\psi)=1 / \mu_{0}$.

If the series (3.2) converges there is an infinite, one-parameter family of $Q$-processes, which includes two members - the minimal and the maximal $Q$-process - with matrices of transition 
functions $P^{\min }(\cdot)$ and $P^{\max }(\cdot)$ that are uniquely defined by the requirement that any $Q$-process with matrix of transition functions $P(\cdot)$ satisfies

$$
P^{\min }(t) \leq P(t) \leq P^{\max }(t), \quad i, j \in \mathcal{N}, \quad t \geq 0,
$$

where $\leq$ denotes componentwise inequality. After introducing duality for birth-death processes in the next subsection we will able to identify the parameter characterizing the individual $Q$ processes.

Given the birth rates $\lambda_{n}$ and death rates $\mu_{n}$ of $\mathcal{X}$ we can define positive numbers $c_{n}$ and $d_{n}$ by (1.2) and, subsequently, polynomials $P_{n}$ by the recurrence relation (1.1). By Favard's theorem the polynomials $P_{n}$ are orthogonal with respect to a finite positive Borel measure on the real axis (with finite moments of all positive orders), and it is shown in [16] and [8] that, in fact, there is such a measure with support on the nonnegative real axis. As before we will assume that the measure is normalized to be a probability measure. So we conclude that a set of birth and death rates uniquely defines a natural measure on the nonnegative real axis, thus confirming the first part of Fact 1.4.

Actually, the natural measure that is defined by the rates $\lambda_{n}$ and $\mu_{n}$ - and hence by the matrix $Q$ - is precisely the measure $\psi$ appearing in Karlin and McGregor's [16] spectral representation for the transition functions of the unique (if the series (3.2) diverges) or minimal (if the series (3.2) converges) $Q$-process, namely,

$$
p_{i j}(t)=(-1)^{i+j} \prod_{k=1}^{j} \frac{1}{\lambda_{k-1} \mu_{k}} \int_{0}^{\infty} e^{-x t} P_{i}(x) P_{j}(x) \psi(d x), \quad i, j \in \mathcal{N}, \quad t \geq 0,
$$

where an empty product is defined to be 1 . If the series (3.2) converges the representation (3.3) still holds for any $Q$-process, provided $\psi$ is replaced by the appropriate $N$-extremal solution of the Stieltjes moment problem associated with the rate set. If $\mu_{0}=0$ every $N$-extremal measure $\psi_{\xi}, 0 \leq \xi \leq \xi_{1}$, corresponds to a birth-death process. The $N$-extremal measures corresponding to a birth-death process with $\mu_{0}>0$ will be identified in the next subsection. In any case, the preceding remarks confirm Fact 1.3.

For completeness' sake we recall from Section 1 that a natural measure on the nonnegative real axis corresponds to an infinite family of rate sets, indexed by the value of $\mu_{0}$, if (and only if) the measure has a finite moment of order -1 .

\subsection{Dual birth-death processes}

Our point of departure in this subsection is a birth-death process $\mathcal{X}$ that is uniquely defined by its birth rates $\lambda_{n}$ and death rates $\mu_{n}$, where $\mu_{0}>0$. Following Karlin and McGregor [17, Section 6], we define the process $\mathcal{X}^{d}$ to be a birth-death process on $\mathcal{N}$ with birth rates $\lambda_{n}^{d}$ and death rates $\mu_{n}^{d}$ given by $\mu_{0}^{d}:=0$ and

$$
\lambda_{n}^{d}:=\mu_{n}, \quad \mu_{n+1}^{d}:=\lambda_{n}, \quad n \geq 0 .
$$

Accordingly, we let

$$
\pi_{0}^{d}:=1 \quad \text { and } \quad \pi_{n}^{d}:=\frac{\lambda_{0}^{d} \lambda_{1}^{d} \cdots \lambda_{n-1}^{d}}{\mu_{1}^{d} \mu_{2}^{d} \cdots \mu_{n}^{d}}=\frac{\mu_{0} \mu_{1} \cdots \mu_{n-1}}{\lambda_{0} \lambda_{1} \cdots \lambda_{n-1}}, \quad n \geq 1,
$$

and note that

$$
\pi_{n+1}^{d}=\mu_{0}\left(\lambda_{n} \pi_{n}\right)^{-1} \quad \text { and } \quad\left(\lambda_{n}^{d} \pi_{n}^{d}\right)^{-1}=\mu_{0}^{-1} \pi_{n}, \quad n \geq 0 .
$$


Hence divergence of the series (3.2) is equivalent to divergence of the series

$$
\sum_{n=0}^{\infty}\left(\pi_{n}^{d}+\frac{1}{\lambda_{n}^{d} \pi_{n}^{d}}\right),
$$

so that $\mathcal{X}^{d}$ is uniquely defined by its rates if and only if $\mathcal{X}$ is uniquely defined by its rates. So within the setting of birth-death processes that are uniquely defined by their rates, the mapping (3.4) establishes a one-to-one correspondence between processes with $\mu_{0}=0$ and those with $\mu_{0}>0$. The processes $\mathcal{X}$ and $\mathcal{X}^{d}$ are therefore called each other's dual.

The transition functions of $\mathcal{X}^{d}$ satisfy a representation formula analogous to (3.3), involving birth-death polynomials $P_{n}^{d}$ and a unique natural probability measure $\psi^{d}$ on the nonnegative real axis with respect to which the polynomials $P_{n}^{d}$ are orthogonal. Still assuming divergence of (3.2) (and hence of (3.5)), we have, by [16, Lemma 3],

$$
\psi^{d}([0, x])=1-\mu_{0} m_{-1}(\psi)+\mu_{0} \int_{[0, x]} y^{-1} \psi(d y), \quad x \geq 0
$$

where $\psi$ is the (natural) measure defined by $\mathcal{X}$ (which must have $m_{-1}(\psi)<\infty$ since $\mu_{0}>0$ ). With $\xi_{i}^{d}$ and $\sigma^{d}$ denoting the quantities defined by (2.4), (2.5) and (2.6) if we replace $\psi$ by $\psi^{d}$, we thus have $\sigma^{d}=\sigma$,

$$
\xi_{1}^{d}=0 \quad \text { and } \quad \xi_{i+1}^{d}=\xi_{i}, i>1, \quad \text { if } \quad \mu_{0} m_{-1}(\psi)<1,
$$

and

$$
\xi_{i}^{d}=\xi_{i}, \quad i \geq 1, \quad \text { if } \quad \mu_{0} m_{-1}(\psi)=1
$$

Interestingly, with $p_{i j}^{d}(t)$ denoting the transition functions of the dual process, we also have

$$
\sum_{j \geq k} p_{i j}^{d}(t)=\sum_{j<i} p_{k-1, j}(t), \quad i, k \in \mathcal{N}, \quad t \geq 0
$$

provided the summations are interpreted to include probability mass, if any, having escaped from $\mathcal{N}$ to the absorbing state -1 or to infinity (see [22] and the references there for details). This property makes duality a useful tool in the analysis of birth-death processes (see, for example, [23]).

If the series (3.2) (and hence the series (3.5)) converges, the situation is more complicated since the rate sets $\left\{\lambda_{n}, \mu_{n}\right\}$ and $\left\{\lambda_{n}^{d}, \mu_{n}^{d}\right\}$ are associated with infinite families of birth-death processes. The following facts have been established in [22]. First, there is the separation result

$$
0<\xi_{i}^{d}<\xi_{i}<\xi_{i+1}^{d}, \quad i \geq 1
$$

where $\xi_{i}$ and $\xi_{i}^{d}$ now represent the spectral points of the natural measures $\psi=\psi_{\xi_{1}}$ and $\psi^{d}=\psi_{\xi_{1}^{d}}^{d}$ that are uniquely defined by the rate sets $\left\{\lambda_{n}, \mu_{n}\right\}$ and $\left\{\lambda_{n}^{d}, \mu_{n}^{d}\right\}$, respectively. (Recall that $\sigma=\sigma^{d}=\infty$.)

Secondly, the $N$-extremal measure $\psi_{\xi}$ is associated with a birth-death process (in the sense of Section 3.1) if and only if $\xi_{1}^{d} \leq \xi \leq \xi_{1}$, while the $N$-extremal solution $\psi_{\xi}^{d}$ is associated with a birth-death process for all $\xi$ satisfying $0 \leq \xi \leq \xi_{1}^{d}$. The birth-death processes associated with the $N$-extremal solutions $\psi_{\xi_{1}}=\psi$ and $\psi_{\xi_{1}^{d}}^{d}=\psi^{d}$ are minimal processes, whereas the birth-death processes corresponding to the $N$-extremal solutions $\psi_{\xi_{1}^{d}}$ and $\psi_{0}^{d}$ are maximal processes. 
Thirdly, (3.6) (and (3.7)) remain valid if (and only if) either $\psi^{d}$ is replaced by $\psi_{0}^{d}$ or $\psi$ by $\psi_{\xi_{1}^{d}}$, that is, we have

$$
\psi_{0}^{d}([0, x])=1-\mu_{0} m_{-1}(\psi)+\mu_{0} \int_{[0, x]} y^{-1} \psi(d y), \quad x \geq 0
$$

and

$$
\psi^{d}([0, x])=1-\mu_{0} m_{-1}\left(\psi_{\xi_{1}^{d}}\right)+\mu_{0} \int_{[0, x]} y^{-1} \psi_{\xi_{1}^{d}}(d y), \quad x \geq 0 .
$$

It follows that the duality concept for rate sets can be extended to birth-death processes also if they are not uniquely defined by their rates, provided one restricts oneself to minimal and maximal processes, and links a minimal process to a maximal process.

We finally remark that a probabilistic interpretation of minimal and maximal processes involves the character of the boundary at infinity (which is not specified by the rates). This boundary may be completely absorbing (the minimal process), completely reflecting (the maximal process), or something in between. Evidently, the distinction is relevant only if the process can explode, that is, reach infinity in finite time.

\subsection{Similar birth-death processes}

Consider, besides the birth-death process $\mathcal{X}$ of Section 3.1, another birth-death process $\tilde{\mathcal{X}}$, with birth rates $\tilde{\lambda}_{n}$ and death rates $\tilde{\mu}_{n}$, coefficients $\tilde{\pi}_{n}$ and transition functions $\tilde{p}_{i j}(\cdot)$. The processes $\mathcal{X}$ and $\tilde{\mathcal{X}}$ are said to be similar if there are constants $c_{i j}, i, j \in \mathcal{N}$, such that

$$
\tilde{p}_{i j}(t)=c_{i j} p_{i j}(t), \quad i, j \in \mathcal{N}, \quad t \geq 0 .
$$

The next theorem shows that, under certain regularity conditions, similarity imposes strong restrictions on the birth and death rates.

Theorem 3.1. Let the birth-death processes $\mathcal{X}$ and $\tilde{\mathcal{X}}$ be either uniquely determined by their rates or minimal. If $\mathcal{X}$ and $\tilde{\mathcal{X}}$ are similar, then their birth and death rates are related as

$$
\tilde{\lambda}_{n}+\tilde{\mu}_{n}=\lambda_{n}+\mu_{n}, \quad \tilde{\lambda}_{n} \tilde{\mu}_{n+1}=\lambda_{n} \mu_{n+1}, \quad n \in \mathcal{N},
$$

while their transition functions satisfy

$$
\tilde{p}_{i j}(t)=\sqrt{\frac{\pi_{i} \tilde{\pi}_{j}}{\tilde{\pi}_{i} \pi_{j}}} p_{i j}(t), \quad i, j \in \mathcal{N}, \quad t \geq 0 .
$$

Conversely, if $\mathcal{X}$ and $\tilde{\mathcal{X}}$ are birth-death processes with rates related as in (3.9) then $\mathcal{X}$ and $\tilde{\mathcal{X}}$ are similar.

In the more restricted setting in which $\mathcal{X}$ and $\tilde{\mathcal{X}}$ are uniquely determined by their rates the statements of this theorem were given in [18, Theorems 1 and 2]. Since the additional restrictions are not used in the proof of the necessity of (3.9) for similarity of $\mathcal{X}$ and $\tilde{\mathcal{X}}$, the question remains whether (3.9) is sufficient for similarity of $\mathcal{X}$ and $\tilde{\mathcal{X}}$ when the processes are not uniquely defined by their rates (but minimal). This, however, follows immediately from Karlin and McGregor's representation formula (3.3), since, considering the remarks preceding (3.3), the polynomials and natural measure associated with $\mathcal{X}$ must be identical to those of $\tilde{\mathcal{X}}$ if $(3.9)$ prevails. Interestingly, Fralix [15] recently established a sufficient condition for similarity in the setting of continuous-time Markov chains (conjectured earlier by Pollett [20]), which amounts to (3.9) when applied to birth-death processes. 
On relating the results of Theorem 3.1 to (1.1) and (1.2) we see that a family of similar birth-death processes is characterized by the fact that all members are associated with the same orthogonal polynomial sequence $\left\{P_{n}\right\}$ - and hence with the same (natural) orthogonalizing measure $\psi$ - while each individual member may be characterized by the value of $\mu_{0}$, which can be any number in $\left[0,1 / m_{-1}(\psi)\right]$. So a family of similar birth-death processes has either one member (if $m_{-1}(\psi)=\infty$ ) or infinitely many members (if $m_{-1}(\psi)<\infty$ ). Note that there is always a member in the family with $\mu_{0}=0$, the representative of the family. By [16, equation (2.4), Lemma 6 on p. 527] we have

$$
\mu_{0}=0 \quad \Longrightarrow \quad m_{-1}(\psi)=\sum_{n=0}^{\infty} \frac{1}{\lambda_{n} \pi_{n}},
$$

so to decide whether the representative of a family is the only member of the family is, given the birth and death rates of the representative, a trivial task.

\section{Results}

Having collected all we need, we are ready to draw conclusions. To start with, consider a rate set $\left\{\lambda_{n}, \mu_{n}\right\}$ with $\mu_{0}>0$ and the natural measure $\psi$ that, by Fact 1.4, is defined by this set. Since, by Fact 1.4 again, $m_{-1}(\psi)<\infty$ and $\mu_{0} \leq 1 / m_{-1}(\psi)$, we can choose $a=\left(\mu_{0} m_{-1}(\psi)\right)^{-1}-1 \geq 0$ and thus link the rate set $\left\{\lambda_{n}, \mu_{n}\right\}$ to the measure $\phi^{(a)}$ defined in (1.4) and (1.5).

Let us first assume that the rate set $\left\{\lambda_{n}, \mu_{n}\right\}$ is such that the series (3.2) diverges, whence it corresponds to a unique birth-death process $\mathcal{X}$. Then, by Fact 1.5, there are two possibilities. The first is that $\psi$ is $\operatorname{det}(\mathrm{S})$, in which case, by Theorem $1.1, \phi^{(a)}$ is also $\operatorname{det}(\mathrm{S})$. The second possibility is that $\psi$ is indet $(\mathrm{S})$ and $m_{-1}(\psi)=1 / \mu_{0}$. But then $a=0$, so that, by Theorem 1.1, $\phi^{(a)}=\phi^{(0)}$ is $\operatorname{det}(\mathrm{S})$ again. So, in any case, $\phi^{(a)}$ is $\operatorname{det}(\mathrm{S})$ and hence natural. If $a=0$ it is possible for $\phi^{(a)}$ to define an infinite family of rate sets, but, as agreed upon in Section 2.1, we will always associate with $\phi^{(a)}$ the unique rate set $\left\{\lambda_{n}^{(a)}, \mu_{n}^{(a)}\right\}$ with $\mu_{0}^{(a)}=0$ determined by the parameters in the recurrence relation (2.1) for the shell polynomials $S_{n}^{(a)}$. So we have now linked the rate set $\left\{\lambda_{n}, \mu_{n}\right\}$ with $\mu_{0}>0$ to a rate set $\left\{\lambda_{n}^{(a)}, \mu_{n}^{(a)}\right\}$ with $\mu_{0}^{(a)}=0$, which, by Fact 1.5 , uniquely defines a birth-death process $\mathcal{X}^{(a)}$. But on comparing (2.2) and (3.4), we see that

$$
\lambda_{n}^{(a)}=\lambda_{n}^{d} \quad \text { and } \quad \mu_{n}^{(a)}=\mu_{n}^{d}, \quad n \geq 0,
$$

so that, actually, $\mathcal{X}^{(a)}=\mathcal{X}^{d}$, the dual process of $\mathcal{X}$. Indeed, on comparing (1.4) and (1.5) with (3.6), we also observe that $\phi^{(a)}=\psi^{d}$, as required. We summarize our findings in the next theorem.

Theorem 4.1. Let $\left\{\lambda_{n}, \mu_{n}\right\}$ with $\mu_{0}>0$ be a rate set for which the series (3.2) diverges, $\mathcal{X}$ the birth-death process defined by this set, and $\psi$ the corresponding measure. Then $\phi^{(a)}$, defined by (1.4) and (1.5), is $\operatorname{det}(\mathrm{S})$ for all $a \geq 0$, while, for $a=\left(\mu_{0} m_{-1}(\psi)\right)^{-1}-1$, it is the measure corresponding to $\mathcal{X}^{d}$, the dual process of $\mathcal{X}$.

Note that the (not so obvious) condition $\mu_{0} m_{-1}(\psi) \leq 1$, which a rate set associated with the natural measure $\psi$ with $m_{-1}(\psi)<\infty$ should satisfy, has a very natural counterpart for the measure of the dual process, namely $a \geq 0$.

Evidently, (3.4) establishes a one-to-one correspondence between rate sets with $\mu_{0}=0$ and those with $\mu_{0}>0$. So, as long as we work in the setting of rate sets that uniquely define a birth-death process, the above procedure mapping the rate set $\left\{\lambda_{n}, \mu_{n}\right\}$ with $\mu_{0}>0$ to the rate set $\left\{\lambda_{n}^{(a)}, \mu_{n}^{(a)}\right\}$ with $\mu_{0}^{(a)}=0$, via the corresponding birth-death processes, must reflect this correspondence. In other words, every measure $\phi$ that corresponds to a rate set with $\mu_{0}=0$, 
must be of the form $\phi^{(a)}$ of (1.4) and (1.5) for some $a \geq 0$, with $\psi$ being the measure of the dual process. Here are the details of this correspondence.

Consider a rate set $\left\{\tilde{\lambda}_{n}, \tilde{\mu}_{n}\right\}$ with $\tilde{\mu}_{0}=0$ for which the analogue of the series (3.2) diverges. Let $\tilde{\mathcal{X}}$ be the birth-death process uniquely defined by this rate set, $\tilde{P}_{n}$ the corresponding polynomials and $\tilde{\phi}$ the corresponding measure, which, in view of the analogue of $(2.7)$, must be $\operatorname{det}(\mathrm{S})$. Then, letting

$$
a=\frac{\tilde{\phi}(\{0\})}{1-\tilde{\phi}(\{0\})} \quad \text { and } \quad \phi^{(0)}=\frac{\tilde{\phi}-\tilde{\phi}(\{0\}) \delta_{0}}{1-\tilde{\phi}(\{0\})}
$$

$\tilde{\phi}$ can be represented as

$$
\tilde{\phi}=\frac{1}{a+1}\left(a \delta_{0}+\phi^{(0)}\right) .
$$

Defining the (probability) measure $\psi$ by

$$
\psi([0, x])=\frac{1}{m_{1}\left(\phi^{(0)}\right)} \int_{[0, x]} y \phi^{(0)}(d y), \quad x \geq 0,
$$

we can apply some results of Berg and Thill $[4,5]$ to conclude the following.

Lemma 4.2. The measure $\psi$ defined by (4.1) is the natural solution of the corresponding moment problem.

Proof. If $\tilde{\phi}(\{0\})>0$ then, by [5, Lemma 5.4], $\psi$ must be $\operatorname{det}(\mathrm{S})$, and hence natural, since $\tilde{\phi}$ is $\operatorname{det}(\mathrm{S})$. If $\tilde{\phi}(\{0\})=0$ (so that $\left.\tilde{\phi}=\tilde{\phi}^{(0)}\right)$ and $\psi$ is $\operatorname{indet}(\mathrm{S})$, then, by [4, Theorem 2.4], the density index of $\tilde{\phi}^{(0)}$ (the largest $n \in \mathbb{N}$ such that the polynomials are dense in $x^{n} \tilde{\phi}^{(0)}(d x)$ ) equals 2 , implying that $\psi$ has density index 1 . Hence, by [4, Theorem 2.1], $\psi$ must be natural. Since

Evidently, $m_{-1}(\psi)<\infty$, so we see that $\psi$ has the properties imposed on $\psi$ in Section 1 .

$$
\int_{[0, \infty)} \tilde{P}_{1}(x) \tilde{\phi}(d x)=\int_{[0, \infty)}\left(x-\tilde{\lambda}_{0}\right) \tilde{\phi}(d x)=0,
$$

we have $m_{1}(\tilde{\phi})=\tilde{\lambda}_{0}$, while

$$
\psi([0, x])=\frac{1}{m_{1}(\tilde{\phi})} \int_{[0, x]} y \tilde{\phi}(d y)=\frac{1}{\tilde{\lambda}_{0}} \int_{[0, x]} y \tilde{\phi}(d y), \quad x \geq 0,
$$

so that $m_{-1}(\psi)=(1-\tilde{\phi}(\{0\})) / \tilde{\lambda}_{0}$. We can now associate a rate set $\left\{\lambda_{n}, \mu_{n}\right\}$ with $\psi$ by letting

$$
\mu_{0}=\frac{1}{(a+1) m_{-1}(\psi)}=\tilde{\lambda}_{0}
$$

so that $0<\mu_{0} \leq 1 / m_{-1}(\psi)$, and choosing $\lambda_{n}$ and $\mu_{n}$ such that the polynomials $P_{n}$ defined by (1.1) and (1.2) are orthogonal with respect to $\psi$. Next identifying $\tilde{\phi}$ with the measure $\phi^{(a)}$ defined in (1.4) and (1.5), we can identify the rates $\tilde{\lambda}_{n}$ and $\tilde{\mu}_{n}$ with the rates $\lambda_{n}^{(a)}$ and $\mu_{n}^{(a)}$, respectively, appearing in the recurrence relation (2.1) for the shell polynomials corresponding to the sequence $\left\{P_{n}\right\}$. On comparing (2.2) and (3.4), we thus find

$$
\tilde{\lambda}_{n}=\lambda_{n}^{d} \quad \text { and } \quad \tilde{\mu}_{n}=\mu_{n}^{d}, \quad n \geq 0 .
$$

It follows that the series (3.5), and hence the series (3.2), diverges, so that the rate set $\left\{\lambda_{n}, \mu_{n}\right\}$ defines a unique birth-death process $\mathcal{X}$. Moreover, $\tilde{\mathcal{X}}=\mathcal{X}^{d}$, the dual process of $\mathcal{X}$. In summary, we can state the converse of Theorem 4.1 as follows. 
Theorem 4.3. Let $\left\{\tilde{\lambda}_{n}, \tilde{\mu}_{n}\right\}$ with $\tilde{\mu}_{0}=0$ be a rate set for which the analogue of the series (3.2) diverges, $\tilde{\mathcal{X}}$ the birth-death process defined by this set, and $\tilde{\phi}$ the corresponding measure. Then, letting $a=\tilde{\phi}(\{0\}) /(1-\tilde{\phi}(\{0\}))$, the measure $\tilde{\phi}$ can be identified with $\phi^{(a)}$, defined by (1.4) and (1.5), where $\psi$ is the natural measure corresponding to a birth-death process $\mathcal{X}$ with $\mu_{0}=$ $\left((a+1) m_{-1}(\psi)\right)^{-1}>0$. Also, $\tilde{\mathcal{X}}=\mathcal{X}^{d}$, the dual process of $\mathcal{X}$.

Still residing in the setting of birth-death processes that are uniquely defined by their rates, we recall from Section 3.3 that a collection of birth-death processes sharing the same natural measure $\psi$ with finite moment of order -1 is called a family of similar processes. The individual members of this family are identified by the value of $\mu_{0}$, which may be any value in the interval $0 \leq \mu_{0} \leq 1 / m_{-1}(\psi)$. However, in view of the preceding observations, it seems more appropriate to exclude the process with $\mu_{0}=0$ from this family and view this process as a member of a new family of birth-death processes, which all have $\mu_{0}=0$ and a measure of the type

$$
\frac{1}{a+1}\left(a \delta_{0}+\psi\right)
$$

where $a \geq 0$, the process at hand corresponding to $a=0$.

Defining families of birth-death processes in this way allows us to extend the duality concept for individual birth-death processes to families of birth-death processes. Indeed, if $\psi_{1}$ is a natural measure with $m_{-1}\left(\psi_{1}\right)<\infty$ then there is a one-to-one correspondence between the family of similar processes with measure $\psi_{1}$ and $\mu_{0}>0$, and the family of processes with $\mu_{0}=0$ and a measure of the type (4.2), where $a \geq 0$ and $\psi \equiv \psi_{2}$ is given by

$$
\psi_{2}([0, x])=\frac{1}{m_{-1}\left(\psi_{1}\right)} \int_{[0, x]} y^{-1} \psi_{1}(d y), \quad x \geq 0,
$$

in the sense that corresponding processes are each other's dual. Note that $\psi_{2}$ is $\operatorname{det}(\mathrm{S})$ by Theorem 1.1. If $m_{-1}\left(\psi_{2}\right)<\infty$, or, equivalently, $m_{-2}\left(\psi_{1}\right)<\infty$, we can view $\psi_{2}$ as the producer of a family of similar birth-death processes with $\mu_{0}>0$, which, in turn, is dual to a family of processes with $\mu_{0}=0$ and a measure of the type (4.2), etc.

Moving beyond the setting of birth-death processes that are uniquely defined by their rates the situation becomes more complicated, but as noted in Section 3.2 the duality concept for rate sets can be extended, provided one restricts oneself to minimal and maximal processes, and links a minimal process to a maximal process. We next elaborate on how this affects the measures involved.

So consider again a rate set $\left\{\lambda_{n}, \mu_{n}\right\}$ with $\mu_{0}>0$, and the natural measure $\psi$ defined by this set. As before we can choose $a=\left(\mu_{0} m_{-1}(\psi)\right)^{-1}-1 \geq 0$ and thus link the rate set $\left\{\lambda_{n}, \mu_{n}\right\}$ to the measure $\phi^{(a)}$ defined in (1.4) and (1.5). We now assume that the rate set $\left\{\lambda_{n}, \mu_{n}\right\}$ is such that the series (3.2) converges, so that, by Fact $1.5, \phi^{(a)}$ is $\operatorname{indet}(\mathrm{S})$ and $a>0$. Subsequently comparing (1.5) and (3.8) we conclude that we actually have $\phi^{(a)}=\psi_{0}^{d}$. It follows that $\phi^{(a)}$ is $N$-extremal (as noted already in [3]) and corresponds to the maximal birth-death process associated with the rate set $\left\{\lambda_{n}^{d}, \mu_{n}^{d}\right\}$ with $\mu_{0}^{d}=0$, given by (3.4). Summarizing we can state the following.

Theorem 4.4. Let $\left\{\lambda_{n}, \mu_{n}\right\}$ with $\mu_{0}>0$ be a rate set for which the series (3.2) converges, $\mathcal{X}$ the minimal birth-death process defined by this set, and $\psi$ the corresponding measure. Then $\mu_{0}<1 / m_{-1}(\psi)$, and $\phi^{(a)}$, defined by (1.4) and (1.5), is $\operatorname{indet(S)}$ for $a>0$. Moreover, for $a=\left(\mu_{0} m_{-1}(\psi)\right)^{-1}-1, \phi^{(a)}$ is the measure corresponding to $\mathcal{X}^{d}$, the maximal process that is dual to $\mathcal{X}$, and hence $N$-extremal.

Remark 4.5. The argument given in [3] for the fact that $\phi^{(a)}$ is $N$-extremal is not entirely clear, but, in any case, a reference to [2, Theorem 8] (besides the reference to [5, Theorem 5.5] given in [3]) is sufficient to justify the statement. 
Of course there is a converse to Theorem 4.4 - the analogue of Theorem 4.3 - which, however, we will not formulate explicitly. It may be more interesting to look at the minimal process corresponding to the rate set $\left\{\tilde{\lambda}_{n}, \tilde{\mu}_{n}\right\}$ with $\tilde{\mu}_{0}=0$ which does not uniquely define a birth-death process, since the associated measure is natural. We give the result without proof and refrain again from formulating its converse explicitly.

Theorem 4.6. Let $\left\{\tilde{\lambda}_{n}, \tilde{\mu}_{n}\right\}$ with $\tilde{\mu}_{0}=0$ be a rate set for which the analogue of the series (3.2) converges, $\tilde{\mathcal{X}}$ the minimal birth-death process defined by this set, and $\tilde{\phi}$ the corresponding measure. Then, letting $a=\tilde{\phi}(\{0\}) /(1-\tilde{\phi}(\{0\}))$, the measure $\tilde{\phi}$ can be identified with $\phi^{(a)}$, defined by (1.4) and (1.5), where $\psi$ is the (natural) measure corresponding to a maximal birth-death process $\mathcal{X}$ with $\mu_{0}=\left((a+1) m_{-1}(\psi)\right)^{-1}>0$, and hence $N$-extremal. Also, $\tilde{\mathcal{X}}=\mathcal{X}^{d}$, the dual process of $\mathcal{X}$.

We finally remark that Theorems 4.1 and 4.4 augment the information given in [16, Lemma 2], while Theorems 4.3 and 4.6 elaborate on [16, Lemma 3].

\section{References}

[1] Anderson W.J., Continuous-time Markov chains. An applications-oriented approach, Springer Series in Statistics: Probability and its Applications, Springer-Verlag, New York, 1991.

[2] Berg C., Christensen J.P.R., Density questions in the classical theory of moments, Ann. Inst. Fourier (Grenoble) 31 (1981), 99-114.

[3] Berg C., Christiansen J.S., A question by T.S. Chihara about shell polynomials and indeterminate moment problems, J. Approx. Theory 163 (2011), 1449-1464, arXiv:1102.2723.

[4] Berg C., Thill M., A density index for the Stieltjes moment problem, in Orthogonal Polynomials and their Applications (Erice, 1990), IMACS Ann. Comput. Appl. Math., Vol. 9, Baltzer, Basel, 1991, 185-188.

[5] Berg C., Thill M., Rotation invariant moment problems, Acta Math. 167 (1991), 207-227.

[6] Berg C., Valent G., The Nevanlinna parametrization for some indeterminate Stieltjes moment problems associated with birth and death processes, Methods Appl. Anal. 1 (1994), 169-209.

[7] Berg C., Valent G., Nevanlinna extremal measures for some orthogonal polynomials related to birth and death processes, J. Comput. Appl. Math. 57 (1995), 29-43.

[8] Chihara T.S., Chain sequences and orthogonal polynomials, Trans. Amer. Math. Soc. 104 (1962), 1-16.

[9] Chihara T.S., On determinate Hamburger moment problems, Pacific J. Math. 27 (1968), 475-484.

[10] Chihara T.S., An introduction to orthogonal polynomials, Mathematics and its Applications, Vol. 13, Gordon and Breach Science Publishers, New York - London - Paris, 1978.

[11] Chihara T.S., Indeterminate symmetric moment problems, J. Math. Anal. Appl. 85 (1982), 331-346.

[12] Chihara T.S., The parameters of a chain sequence, Proc. Amer. Math. Soc. 108 (1990), 775-780.

[13] Chihara T.S., Shell polynomials and indeterminate moment problems, J. Comput. Appl. Math. 133 (2001), 680-681.

[14] Coolen-Schrijner P., van Doorn E.A., Orthogonal polynomials on $\mathbb{R}^{+}$and birth-death processes with killing, in Difference Equations, Special Functions and Orthogonal Polynomials, Editors S. Elaydi, J. Cushing, R. Lasser, A. Ruffing, V. Papageorgiou, W. Van Assche, World Sci. Publ., Hackensack, NJ, 2007, 726-740.

[15] Fralix B., When are two Markov chains similar?, Statist. Probab. Lett. 107 (2015), 199-203.

[16] Karlin S., McGregor J.L., The differential equations of birth-and-death processes, and the Stieltjes moment problem, Trans. Amer. Math. Soc. 85 (1957), 489-546.

[17] Karlin S., McGregor J., The classification of birth and death processes, Trans. Amer. Math. Soc. 86 (1957), 366-400.

[18] Lenin R.B., Parthasarathy P.R., Scheinhardt W.R.W., van Doorn E.A., Families of birth-death processes with similar time-dependent behaviour, J. Appl. Probab. 37 (2000), 835-849.

[19] Pedersen H.L., Stieltjes moment problems and the Friedrichs extension of a positive definite operator, J. Approx. Theory 83 (1995), 289-307. 
[20] Pollett P.K., Similar Markov chains, J. Appl. Probab. 38A (2001), 53-65.

[21] Shohat J.A., Tamarkin J.D., The problem of moments, Math. Surveys Monogr., Vol. 1, Amer. Math. Soc., New York, 1943.

[22] van Doorn E.A., The indeterminate rate problem for birth-death processes, Pacific J. Math. 130 (1987), 379-393.

[23] van Doorn E.A., Representations for the decay parameter of a birth-death process based on the CourantFischer theorem, J. Appl. Probab. 52 (2015), 278-289.

[24] van Doorn E.A., Spectral properties of birth-death polynomials, J. Comput. Appl. Math. 284 (2015), 251258. 